\title{
Two-dimensional complex parity-time-symmetric photonic structures
}

\author{
M. Turduev, ${ }^{1}$ M. Botey, ${ }^{2}$ I. Giden, ${ }^{1}$ R. Herrero, ${ }^{2}$ H. Kurt, ${ }^{1}$ E. Ozbay, ${ }^{3}$ and K. Staliunas ${ }^{2,4}$ \\ ${ }^{1}$ Department of Electrical and Electronics Engineering, TOBB University of Economics and Technology, 06560 Ankara, Turkey \\ ${ }^{2}$ DONLL, Departament de Física i Enginyeria Nuclear, Universitat Politècnica de Catalunya (UPC), Edifici Gaia, \\ Rambla Sant Nebridi 22, 08222 Terrassa, Spain \\ ${ }^{3}$ Department of Physics and Department of Electrical and Electronics Engineering, Nanotechnology Research Center, \\ Bilkent University, 06800 Ankara, Turkey \\ ${ }^{4}$ Institució Catalana de Recercai Estudis Avançats (ICREA), Passeig Lluis Companys 23, 08010 Barcelona, Spain
}

(Received 24 June 2014; published 20 February 2015)

\begin{abstract}
We propose a simple realistic two-dimensional complex parity-time-symmetric photonic structure that is described by a non-Hermitian potential but possesses real-valued eigenvalues. The concept is developed from basic physical considerations to provide asymmetric coupling between harmonic wave components of the electromagnetic field. The structure results in a nonreciprocal chirality and asymmetric transmission between inand out-coupling channels into the structure. The analytical results are supported by a numerical study of the Bloch-like mode formations and calculations of a realistic planar semiconductor structure.
\end{abstract}

DOI: 10.1103/PhysRevA.91.023825

PACS number(s): 42.70.Qs, 78.20.Bh, 81.05.Fb, 85.60.-q

\section{INTRODUCTION}

Parity-time (PT) -symmetric structures were initially proposed as exotic systems with unusual properties; despite their complex-valued potentials, the non-Hermitian Hamiltonians describing those systems can have real eigenvalues [1]. First regarded as a curiosity in quantum mechanics, such systems have recently been shown to have interesting and useful applications in classical wave systems, especially in optics. Indeed, PT-symmetric photonic systems have shown intriguing new features, such as PT phase transitions [2] and the realization of unidirectional invisible media $[3,4]$ or unidirectional waveguide transmitters [5,6]; some of these effects have already been realized experimentally $[2,5,6]$.

PT symmetry requires that the complex potential, $U(\vec{r})=$ $U^{\operatorname{Re}}(\vec{r})+i U^{\operatorname{Im}}(\vec{r})$, obey the symmetry requirement $U(\vec{r})=$ $U^{*}(-\vec{r})$, which means that the real part of the potential is an even function, $U^{\operatorname{Re}}(\vec{r})=U^{\operatorname{Re}}(-\vec{r})$, whereas the imaginary part is odd, $U^{\operatorname{Im}}(\vec{r})=-U^{\operatorname{Im}}(-\vec{r})$. Although the imaginary part of the potential is generally difficult to obtain in nature, this is not the case in optics. The classical analog to the real part of the potential in optics is the refractive index, and the gain-loss is analogous to its imaginary part. Therefore, by combining the index and gain-loss modulations with the required symmetries, such optical systems become classical analogs of quantum systems described by PT-symmetric Hamiltonians [2-6].

To date, the pioneering works referenced above and recent extensive literature on optical PT symmetry cover mostly onedimensional (1D) systems. On the other hand, recent works on systems with gain-loss modulations in two dimensions $[7,8]$, and also on complex two-dimensional $2 \mathrm{D}$ crystals $[9,10]$ where the gain-loss and index are simultaneously modulated, have shown the micro- and nanophotonics to be a platform for developing synthetic materials with novel beam propagation effects. However, none of these cases [7-10] can be attributed to PT-symmetric systems because they do not meet the requirements of PT symmetry.

In this paper, we propose a 2D PT-symmetric complex photonic structure, and we show the properties inherent to its $2 \mathrm{D}$ character. We explore the light propagation within it, both by realistic numerical calculations using the finite-difference time-domain (FDTD) method, and by analyzing the Bloch-like modes due to the complex modulation of the potential. We observe strong asymmetric clockwise-counterclockwise flows of light in the Bloch-like modes close to the crystallographic resonances or, equivalently, close to high-symmetry points. As a basic effect, we numerically show the measurable asymmetric transmission of a Gaussian light beam incident on a finite-sized structure resulting from asymmetric wave coupling.

\section{DERIVATION OF THE 2D HONEYCOMB PT-SYMMETRIC STRUCTURE}

To introduce the coupling effects in a 2D PT-symmetric photonic structure, we start from a 1D PT-symmetric optical system, the properties of which are summarized in Fig. 1. This is essentially a superposition of a 1D Bragg mirror [Fig. 1(a1)] and a balanced gain-loss modulation with the same periodicity but spatially displaced by a quarter-period [Fig. 1(a2)]. In the simplest case, we can consider the harmonic potential of the structure in the form $n(x)=n[\cos (q x)+i \sin (q x)]$, more conveniently expressed as

$$
n(x)=n \exp (\text { iqx }),
$$

where $q$ is the reciprocal-lattice vector of the modulation, and $n$ is the amplitude of the complex index modulation. Clearly, such a modulation unidirectionally couples a wave with wave vector $k_{B}$ to $k_{A}=k_{B}+q$. In the right column of Fig. 1(a2), a left-propagating resonant wave, $k_{B} \approx-q / 2$, is coupled to $k_{A}=k_{B}+q \approx q / 2$ and is thus Bragg-reflected to the right. Alternatively, a harmonic Bragg reflector with real-valued potential,

$$
n(x)=n \cos (q x)=\frac{n}{2}[\exp (i q x)+\exp (-i q x)],
$$

symmetrically couples, at resonance, $k_{A} \approx q / 2$ with $k_{B} \approx$ $-q / 2$, as illustrated in the right column of Fig. 1(a1). In this way, the 1D PT-symmetric modulation given by Eq. (1) breaks the symmetry of left-right wave coupling and propagation, 
(a1)
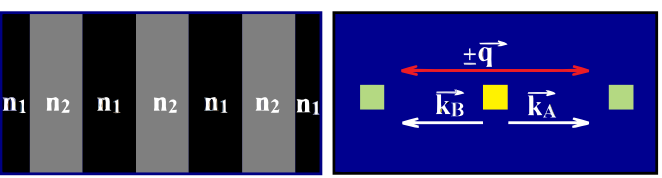

(a2)

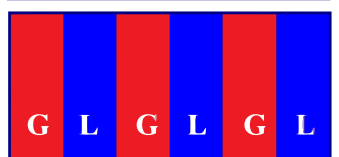

(b1)

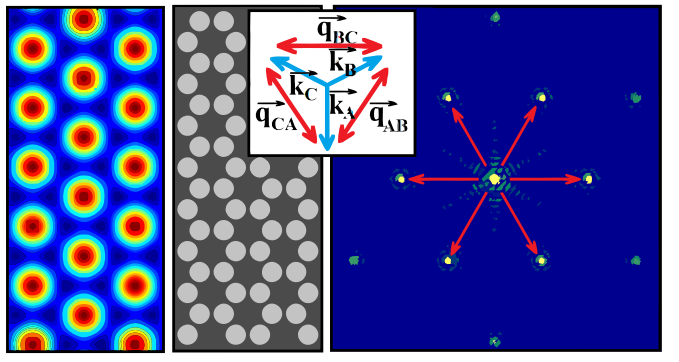

(b2)

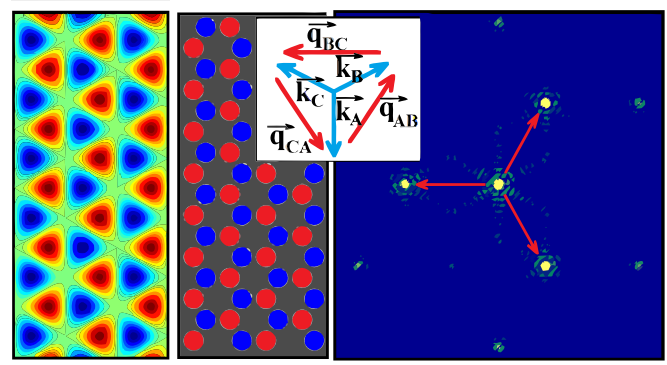

FIG. 1. (Color online) (a1) Left: 1D Bragg reflector. Right: Fourier transform (FT) of the structure, reciprocal-lattice vectors and reciprocal coupling of wave vectors at resonance, $n_{1}>n_{2}$. (a2) Left: Gain-loss distribution ( $G$, red; $L$, blue). Right: FT of the combined 1D PT-symmetric structure from (a1) and (a2), symmetric lattice vectors, and asymmetric coupling at resonance. (b1) Left: Real part of Eq. (3), $n_{0}=1.1, \Delta n=0.1$. Center: Arrangement of cylinders. Right: FT of real cylinder's structure and lattice vectors. (b2) Left: Imaginary part of Eq. (3). Center: Honeycomb arrangement of gain-loss cylinders, $n=1.1 \pm 0.1 i, n_{0}=1.3$. Right: $\mathrm{FT}$ of full 2D PT-symmetric arrangement of cylinders. Insets in (b1) and (b2) show the symmetric and asymmetric coupling at resonance.

which becomes most pronounced at resonance. Note that this symmetry breaking is the main difference between the potentials in Eqs. (1) and (2), and it is the reason for all the peculiarities displayed by PT-symmetric systems, while reciprocity always holds [11].

Keeping this basic principle in mind, we consider the PT-symmetric complex crystal in 2D space. The simplest choice is the trivial extension of the 1D PT symmetry to $2 \mathrm{D}, n(\vec{r})=n_{x} \exp \left(i q_{x} x\right)+n_{y} \exp \left(i q_{y} y\right)$, which simply factorizes the PT symmetries in both quadratures but does not lead to new 2D peculiarities. Therefore, we intend to build the nonfactorizable PT symmetries, i.e., nonfactorizable unidirectional coupling between the plane-wave components, assuming that it will introduce 2D peculiarities (in comparison with 1D PT-symmetric systems).

We chose a triangular lattice as the simplest nontrivial case:

$$
n(\vec{r})=n_{0}+\Delta n \sum_{j=A B, B C, C A} \exp \left(i \vec{q}_{j} \cdot \vec{r}\right)
$$

which is generated by three vectors symmetrically rotated by angles of $2 \pi / 3$ with respect to one another, namely, $\vec{q}_{A B, C A}=$ $(q / 2, \pm q \sqrt{3} / 2)$ and $\vec{q}_{B C}=(-q, 0)$, as represented in the right column of Fig. 1(b2), where $n_{0}$ is the refractive index of the dielectric embedding medium, and $\Delta n$ determines the amplitude of the complex modulation. Note that considering only the real part of Eq. (3) leads to the corresponding dielectric $\mathrm{PhC}$ with sixfold symmetry, as represented by Fig. 1(b1). At resonance, $\left|k_{A, B, C}\right|=q \sqrt{3} / 3$, such a real structure ( $\mathrm{PhC}$ case) reciprocally couples the plane-wave components directed along the symmetry axes, as schematically shown in the inset of Fig. 1(b1). However, for the complex lattice described by Eq. (3), the coupling is analogous to that given by Eq. (1), being PT-symmetric in any direction. Such a complex lattice exhibits a threefold symmetry, as shown in the inset of Fig. 1(b2). This can be expected to produce peculiarities in PT-symmetric systems.

Next, to design a realistic 2D PT-symmetric structure, we replace the lower refractive index areas with low refractive index cylinders [central column in Fig. 1(b1)]. The right column of Fig. 1(b1) displays a sixfold reciprocal space (Fourier transform) of the cylinder arrangement enabling symmetric coupling. However, when such cylinders alternatively exhibit gain and loss, as schematically represented in the central column of Fig. 1(b2), the complex distribution of the index contains the expected PT symmetry. Indeed, the reciprocal space of the arrangement of cylinders [right column of Fig. 1(b2)] reproduces the three points in the configuration proposed in Eq. (3), leading to unidirectional coupling between wave components. Apart from the three points indicating the lattice vectors, $\vec{q}_{A B}, \vec{q}_{B C}$, and $\vec{q}_{C A}$, other higher-order harmonics of the complex distribution appear due to the nonharmonic (stepwise) modulation of the potential.

The triangular lattice is seemingly the simplest nontrivial case of a nonfactorizable 2D PT-symmetric complex crystal. Further nontrivial cases could be realized for higher oddfold rotational symmetry, which would also yield nontrivial 2D PTsymmetric quasicrystals. Here we consider only this triangular case.

\section{ASYMMETRIC CHIRAL EXCITATION}

We numerically check whether the proposed system displays the expected properties of complex PT-symmetric systems, in particular the asymmetric flow of light. Differently from one dimension, the asymmetric coupling between wave vectors rotates the input by $\pm 2 \pi / 3$, depending on the input channel. In other words, the structure is expected to display a type of chiral nonreciprocity. This test is performed numerically using the well-established FDTD technique [12]. We consider two finite-size structures of the same symmetry containing the real and complex distributions shown in Figs. 2(a) and 2(b), respectively.

We first analyze the propagation of a short broadband pulse incident on the structure from the top in the vertical direction and calculate the transmitted intensity on two detectors, symmetrically located on both sides of the structure [T1 and T2 in Figs. 2(a) and 2(b)]. The resulting spectral transmission in the clockwise and counterclockwise directions, normalized to the incident pulse intensity, is represented 

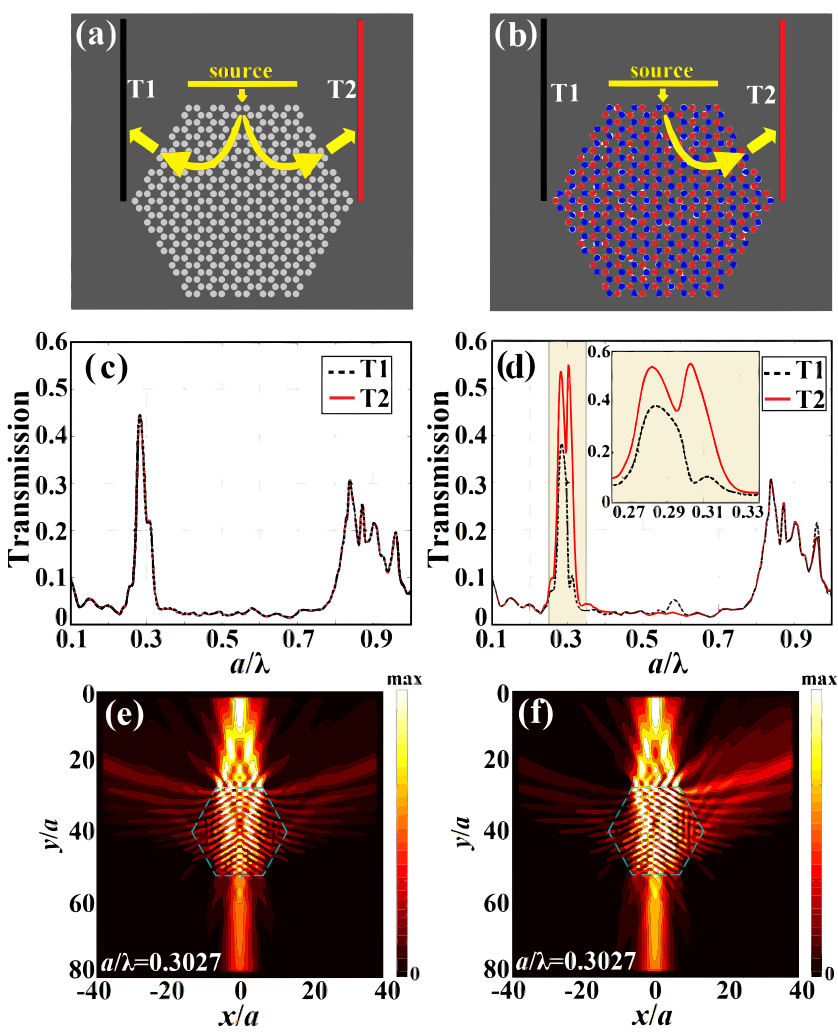

FIG. 2. (Color online) Schematic representation of (a) 2D PhC (real) and (b) 2D PT-symmetric (complex) structures, and (c) and (d) source and detectors. Clockwise and counterclockwise transmissions as a function of frequency, in $a / \lambda$ units, (" $a$ " is the center-to-center distance between cylinders of radius, $R=0.45 a$ ) for structures in (a) and (b), respectively. Inset in (d) is a magnified view within $a / \lambda=0.25-0.35$. (e) and (f) Normalized intensity distributions for an incident Gaussian beam (width 14a) on (a) and (b), respectively. See the supplemental material in Ref. [14].

in Figs. 2(c) and 2(d) for each structure. When comparing the transmissions, we clearly see the expected asymmetry arising precisely at resonant frequencies $a / \lambda \approx 0.3$, where " $a$ " is the center-to-center distance between cylinders; note that $q=4 \pi / 3 a$ [see Figs. 2(c) and2(d)]. Although the T1 and T2 spectra coincide perfectly for all frequencies, for the $\mathrm{PhC}$ case in Fig. 2(c), the counterclockwise (clockwise) transmission is enhanced (reduced) at resonance for the 2D PT-symmetric structure in Fig. 2(d). Note that, except for a higher-order resonance at $a / \lambda \approx 0.6$ (due to high-order mode coupling), the symmetry is still unbroken far from resonance, and both curves coincide well at other frequencies.

For the $\mathrm{PhC}$ structure, an incident wave $k_{A}$ couples symmetrically to $k_{B}$ and $k_{C}$, as schematically shown in the inset of Fig. 1(b1). We can observe that the field distribution depicted in Fig. 2(e), obtained by numerical FDTD simulation, is perfectly symmetric. However, the asymmetric flow of light within the complex system enhances the transmission to the counterclockwise output channel, T2, in Fig. 2(f), whereas transmission to the T1 channel is suppressed. Figure 2(f) demonstrates at a glance the asymmetric coupling schematically represented in the inset of Fig. 1(b2); the incident wave
$\vec{k}_{A}$ is coupled to $\vec{k}_{B}$ but not to $\vec{k}_{C}$. Finally, we also find that the situation depicted in Fig. 2(e) is very similar to the field distribution from the 2D PT-symmetric structure far from resonance, where no symmetry breaking is predicted.

Finally, we note that whereas $\vec{k}_{A}$ couples to $\vec{k}_{B},-\vec{k}_{A}$ couples to $-\vec{k}_{C}$. Thus, a $-\vec{k}_{A}$ wave, incident from the base upward to the structure, would be transmitted clockwise instead of propagating counterclockwise within the structure due to the nonreciprocal chirality of the system. Note that the closed set of lattice vectors $\left(q_{A B}+q_{B C}+q_{C A}=0\right)$ enables the simultaneous resonance of two disjoint triads, namely $\left(k_{A}, k_{B}, k_{C}\right)$ and $\left(-k_{A},-k_{C},-k_{B}\right)$ in a circular chiral coupling. The counterclockwise chiral mode is excited by $k_{A}$, and the clockwise mode by $-k_{A}$, rendering the chiral flow of light input-dependent.

\section{CHIRAL BLOCH-LIKE MODES CLOSE TO THE PT-TRANSITION POINT}

For a $\mathrm{PhC}$, the Bloch modes are defined as localized electromagnetic states of the periodic media that are invariant in propagation. However, in a complex system described by a non-Hermitian Hamiltonian, complex Bloch-like modes may either amplify or decay in time. Below, we calculate such Bloch-like modes analytically considering the simple case of a harmonic PT-symmetric complex crystal of triangular symmetry. We consider an incident plane wave with a polarization perpendicular to the plane of the crystal and a wave vector directed vertically, $\vec{k}=(0,-k)$, near resonance: $\vec{k}=\vec{k}_{A}+\Delta \vec{k}$. The small variations are considered to be in the same incident direction: $\Delta \vec{k}=\left(0,-\Delta k_{y}\right)$. Disregarding the second time derivatives, the wave equation can be written as

$$
-2 i \omega \partial_{t} \vec{E}=\frac{c^{2}}{n(\vec{r})^{2}} \nabla^{2} \vec{E}+\omega^{2} \vec{E} .
$$

We expand the electric field into the first three harmonics of the field, which are resonant in the lattice, namely $\vec{k}_{A}=$ $\left(0,-k_{0}\right), \vec{k}_{B}=\vec{k}_{A}+\vec{q}_{A B}$, and $\vec{k}_{C}=\vec{k}_{A}-\vec{q}_{C A}$, and we obtain, for the TM polarization,

$$
E=\sum_{j=A, B, C} a_{j} \exp \left[i\left(\vec{k}_{j}+\Delta \vec{k}\right) \cdot \vec{r}\right] .
$$

Introducing the expansion in (5) into (4) yields coupled equations between their amplitudes, $a_{A}, a_{B}, a_{C}$,

$$
-i \frac{n_{0}}{k_{0} c} \partial_{t}\left(\begin{array}{l}
a_{A} \\
a_{B} \\
a_{C}
\end{array}\right)=\left(\begin{array}{ccc}
\vec{k}_{A} \cdot \Delta \vec{k} & \Delta n / n_{0} & 0 \\
0 & \vec{k}_{B} \cdot \Delta \vec{k} & \Delta n / n_{0} \\
\Delta n / n_{0} & 0 & \vec{k}_{C} \cdot \Delta \vec{k}
\end{array}\right)\left(\begin{array}{l}
a_{A} \\
a_{B} \\
a_{C}
\end{array}\right) .
$$

The dispersion diagrams, i.e., the temporal eigenvalues and the associated Bloch-like modes, are obtained by diagonalization of the matrix in (6). Figures 3(a) and 3(b) display the real and imaginary parts, respectively, of the matrix eigenvalues for the three Bloch-like modes at the edge of the Brillouin zone, i.e., at resonance between lattice vectors. The temporal evolution of the Bloch mode is defined by the matrix eigenvalues with a factor $i n_{0} / k_{0} c$. As expected, sufficiently 

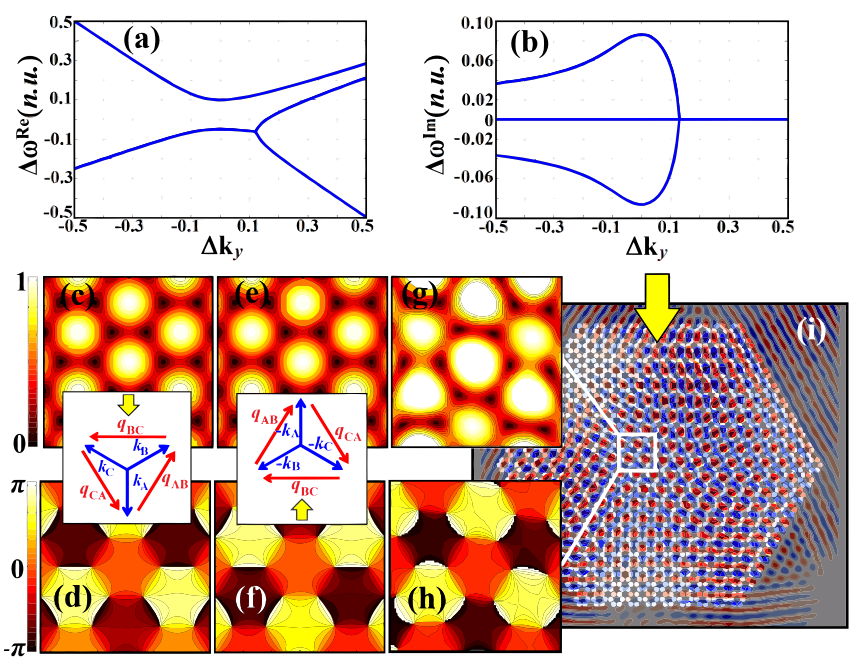

FIG. 3. (Color online) Calculated dispersion diagrams and Bloch modes. (a) Real and (b) imaginary parts of the matrix eigenvalues $\Delta \omega$, where $\Delta k_{y}$, on the horizontal axis, is the distance from resonance in $k_{0}$ units. (c) Amplitude and (d) phase of the amplified chiral Bloch mode for $\Delta k=0$, when illuminated from above and from below (e) and (f), respectively; the insets show the counterclockwise/clockwise asymmetric coupling. (g) Field intensity and (h) phase distribution within the hexagon for an incident Gaussian beam with carrier frequency $a / \lambda=0.303$, corresponding magnified $6 a \times 6 a$ region. (i) Field amplitude obtained directly from FDTD calculation; the arrow indicates the input channel. See the supplemental material in Ref. [14].

far from resonance, all the eigenvalues are real-valued (where the asymmetry of the coupling is not pronounced). Close to resonance, the PT phase transition occurs, and we obtain Bloch modes with complex eigenvalues, one with a negative imaginary part and hence amplified in time. Therefore, in an extended structure, after a finite propagation time, the field distribution is expected to exhibit the amplitude and phase corresponding to this amplified mode. Such an amplitude and phase of the most amplified Bloch mode, as calculated analytically from Eq. (6), are depicted in Figs. 3(c) and 3(d), respectively.

To check the analytic predictions, we analyze the field evolution after excitation by a relatively long Gaussian pulse with central frequency at resonance and the spectrum narrower than the width of the transmission resonance peak in Fig. 2(d). Within the structure (a larger version of the same honeycomb configuration), the incident radiation is redistributed among all the coupled harmonics approaching a stationary distribution of the growing Bloch-like mode, after a sufficiently long time. The analytically calculated amplitude and phase of the amplified chiral Bloch-like mode are shown in Figs. 3(c) and 3(d) [Figs. 3(e) and 3(f)], when the structure is illuminated from above (below), respectively. The result presented in Fig. 3(i) is used to extract the amplitude and phase of the Bloch mode shown in Figs. 3(g) and 3(h), respectively. The results agree well with the analytically calculated amplified Bloch modes. The differences may be attributed mainly to the simplified model used (not accounting for the real shape of the

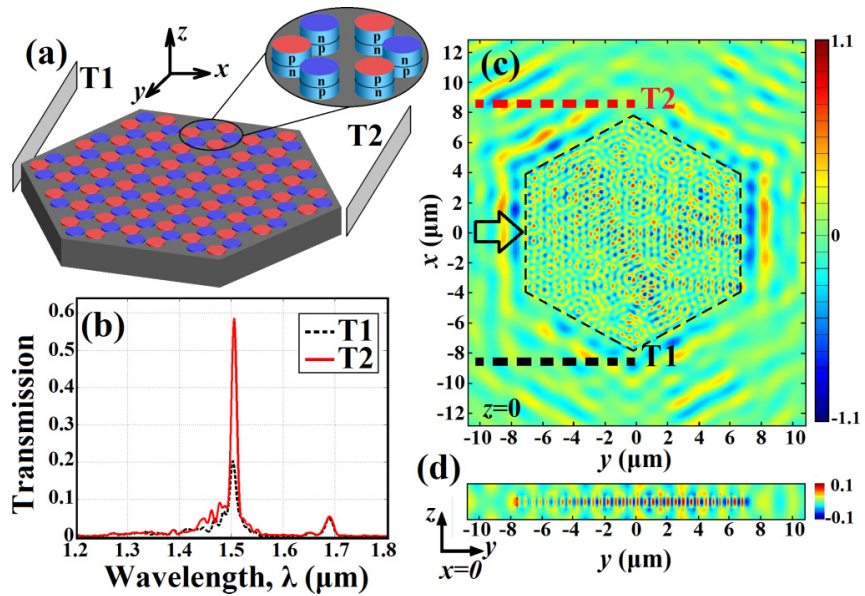

FIG. 4. (Color online) (a) Dielectric slab, $n=3.474,0.612 \mu \mathrm{m}$ high, with holes of radii $0.45 \mu \mathrm{m}$ filled by $p-n / n-p$ semiconductor junctions, $n=3.46 \pm 0.007 i ; a=1.0 \mu \mathrm{m}$, where red (blue) circles indicate gain (loss) areas (b) Clockwise-counterclockwise normalized transmission on detectors $\mathrm{T} 1$ and $\mathrm{T} 2$. Electric field distribution snapshots at cross-sectional planes (c) $z=0$ and (d) $x=0$. The black arrow in (c) indicates the input channel.

scatter) and the interplay between higher-order harmonics, as well as to the finite size of the structure.

\section{IMPLEMENTATION PROPOSAL}

Finally, we propose a possible realization of the investigated 2D PT-symmetric complex structure, which could be implemented and measured in microphotonic devices. The configuration illustrated in Fig. 4(a) consists of a silicon slab with a honeycomb lattice of alternating $p-n$ and $n-p$ semiconductor junctions. Full 3D FDTD numerical simulations were performed using the LUMERICAL software package [13]. The device is illuminated by a broadband pulse with a Gaussian profile, with a source of $7 \mu \mathrm{m}$ width and $0.5 \mu \mathrm{m}$ height. Detectors T1 and T2 are symmetrically placed on either side of the structure as shown in Fig. 4(a) to record the transmission. The calculated normalized transmission spectra at T1 and T2 are depicted in Fig. 4(b). A measurable clockwisecounterclockwise asymmetry is observed in the transmission near resonance at the wavelength $\lambda=1.501 \mu \mathrm{m}$ (wavelength in a vacuum). The steady-state electric-field distributions at the cross-sectional $x y$ plane $(z=0)$ and $y z$ plane $(x=0)$ are shown in Figs. 4(c) and 4(d), respectively. The electric-field snapshot in Fig. 4(c) shows the asymmetric light transmission along the directions of $\mathrm{T} 1$ and $\mathrm{T} 2$ at the resonance frequency. Furthermore, the cross-sectional field distribution depicted in Fig. 4(d) proves the vertical confinement and guiding of the propagating beam inside the slab. As a result, the out-of-plane losses are almost negligible for this specific design.

\section{CONCLUSION}

To conclude, we propose a simple 2D PT-symmetric photonic structure and analyze the propagation of light with it. As predicted, we see that close to resonance, the system exhibits 
a nonreciprocal chirality associated with asymmetric wave coupling between the plane-wave components. Therefore, such a 2D PT-symmetric structure with a hexagonal shape asymmetrically transmits light beams incident on it. In addition, we analytically calculate the Bloch-like mode formations and find that indeed the more amplified mode agrees well with the complex field and phase distributions in the structure at resonance. Following the proposed scheme, we design and numerically analyze, using full 3D FDTD simulations, a 2D PT-symmetric feasible configuration. The proposed 2D planar semiconductor structure could be produced by microfabrication and microstructuration of the electrodes to achieve the modulated gain-loss. It may be expected that new synthetic optical components could rely on such optical systems.

\section{ACKNOWLEDGMENTS}

We acknowledge financial support by Spanish Ministerio de Ciencia e Innovación, the European Union FEDER through project FIS2011-29731-C02-01, Generalitat de Catalunya (2009 SGR 1168), and the Turkish Academy of Science.
[1] C. M. Bender and S. Boettcher, Real spectra in non-Hermitian Hamiltonian having PT symmetry, Phys. Rev. Lett. 80, 5243 (1998).

[2] A. Guo, G. J. Salamo, D. Duchesne, R. Morandotti, M. VolatierRavat, V. Aimez, G. A. Siviloglou, and D. N. Christodoulides, Observation of PT-symmetry breaking in complex optical potentials, Phys. Rev. Lett. 103, 093902 (2009).

[3] S. Longhi, Invisibility in PT-symmetric complex crystals, J. Phys. A 44, 485302 (2011).

[4] Z. Lin, H. Ramezani, T. Eichelkraut, T. Kottos, H. Cao, and D. N. Christodoulides, Unidirectional invisibility induced by PT-symmetric periodic structures, Phys. Rev. Lett. 106, 213901 (2011).

[5] C. E. Ruter, K. G. Makris, R. El-Ganainy, D. N. Christodoulides, M. Segev, and D. Kip, Observation of parity-time symmetry in optics, Nat. Phys. 6, 192 (2010).

[6] L. Feng, Y.-L. Xu, W. S. Fegadolli, M.-H. Lu, J. E. Oliveira, V. R. Almeida, Y.-F. Chen, and A. Scherer, Experimental demonstration of a unidirectional reflectionless parity-time metamaterial at optical frequencies, Nat. Mater. 12, 108 (2012).

[7] K. Staliunas, R. Herrero, and R. Vilaseca, Subdiffraction and spatial filtering due to periodic spatial modulation of the gainloss profile, Phys. Rev. A 80, 013821 (2009).
[8] M. Botey, R. Herrero, and K. Staliunas, Light in materials with periodic gain-loss modulation on a wavelength scale, Phys. Rev. A 82, 013828 (2010).

[9] R. Herrero, M. Botey, M. Radziunas, and K. Staliunas, Beam shaping in spatially modulated broad-area semiconductor amplifiers, Opt. Lett. 37, 5253 (2012).

[10] M. Radziunas, M. Botey R. Herrero, and K. Staliunas, Intrinsic beam shaping mechanism in spatially modulated broad area semiconductor amplifiers, Appl. Phys. Lett. 103, 132101 (2013).

[11] S. Fan, R. Baets, A. Petrov, Z. Yu, J. D. Joannopoulos, W. Freude, A. Melloni, M. Popović, M. Vanwolleghem, D. Jalas, M. Eich, M. Krause, H. Renner, E. Brinkmeyer, and C. R. Doerr, Comment on nonreciprocal light propagation in a Silicon photonic circuit, Science 335, 38 (2012).

[12] Simulations are performed using the open-source software MEEP, initially developed at MIT (http://ab-initio.mit.edu/) for electromagnetic field calculations.

[13] Lumerical FDTD Solutions, Inc., http://www.lumerical.com

[14] See Supplemental Material at http://link.aps.org/supplemental/ 10.1103/PhysRevA.91.023825 for a frequency scan of the distribution showing the PT-phase transitions, and for videos of amplitude and phase evolutions and the counterclockwise flow of light. 